\title{
Application of altimeter experiments of Planet-B orbiter to the exploration of Martian surface and subsurface layers
}

\author{
Tatsuaki Okada ${ }^{1}$ and Takayuki Ono ${ }^{2}$ \\ ${ }^{1}$ Division of Planetary Science, Institute of Space and Astronautical Science, Sagamihara, 229-8510, Japan \\ ${ }^{2}$ Department of Astronomy and Geophysics, Tohoku University, Sendai 980-8578, Japan \\ (Received July 31, 1997; Revised November 21, 1997; Accepted February 12, 1998)
}

\begin{abstract}
Application of altimeter experiment of Planet-B orbiter to the exploration of Martian surface and subsurface layers is planned by using the ALT mode data from the "PWS (Plasma Waves and Sounder)" instrument. The topographic profile along-the-track from the orbiter to the nadir point of Martian surface can be obtained in detail owing to the high accuracy of the PWS altimetry of about $20 \mathrm{~m}$. The characteristics of altimeter echo intensities are also applicable to obtain the electromagnetic properties which were functions of bulk density, humidity, and abundance of ferromagnetic minerals of the uppermost layer of Martian surfaces. The surface macroscopic roughness was also studied since the rise time was slower for the echo from the rougher surface. Another possibility of the application of the altimeter experiment can be given by identifying the subsurface echoes and it is used as a probe for sensing physical properties from tens to hundreds meters below the surface, including possible exploration of liquid water.
\end{abstract}

\section{Introduction}

Physical properties of planetary surfaces depend on the chemical composition and physical conditions influenced by both the endogenous and exogenous processes which have suffered such as volcanisms, tectonics, impact craterings, and meteorological activities. The electromagnetic properties of rocks and soils from the Earth and Moon have been studied and formulated as functions of bulk density, humidity, and abundance of ferromagnetic minerals (Campbell and Ulrichs, 1969; Strangway and Olhoeft, 1977). As was carried out during Apollo 17 mission, HF radio pulses was used to probe the properties and structures of top and sub-surface layers (Phillips et al., 1973). The feasibility of the application of the altimeter to be applied to Martian surface layers in Planet$\mathrm{B}$ mission is considered in this article with special attention to the soil condition and composition, layered structures, amount of water, and geomorphologic roughness.

The Martian surface has a variety of geologic features. The APXS on Sojourner rover of Mars Pathfinder mission measured the elemental composition of rocks and soils, to find a rock called "Barnacle Bill" and "Yogi" with silicic or andesitic composition, while "Scooby Doo" and the background soils are basaltic (Rieder et al., 1997), which is consistent with the Viking XRF results (Clark et al., 1977). This suggests the evidence that the terrestrial-like igneous processes occurred to compose diversity of composition sufficient to produce andesitic rocks, and that at least some local geologic areas consist non-basaltic composition.

Many Martian impact craters have their lobate layered ejecta blankets, origin of which is still controversial but the pattern maybe formed by the reason why the airborne debris

Copy right (C) The Society of Geomagnetism and Earth, Planetary and Space Sciences (SGEPSS); The Seismological Society of Japan; The Volcanological Society of Japan; The Geodetic Society of Japan; The Japanese Society for Planetary Sciences. was saturated with groundwater and tended to flow across the ground as fluid-like mass after ejected outward. The bulk density might be a clue to clarify whether or not liquid water play a key role for the pattern formation.

Southern Amazonis and Hellas planitias are the radar dark areas called "stealth" from which almost no radar echo are detected while Earth-based radar observations are performed at several $\mathrm{cm}$ in wavelength (Muhleman et al., 1991). An alternative explanation is that the uppermost layers are covered with very soft, porous regolith whose dielectric constant equals approximately to unity while the very flat and compacted surfaces reflect the radar specularly and never return it backward to the Earth.

Based on the deformed morphologic features associated with terrestrial polar regions, the upper surface layers are considered frozen and saturated with ices in the mid-latitude to polar region while highly desiccated in the equatorial region. The electromagnetic properties of ice-saturated layers are obviously different from those of regolith or rocky layers (Olhoeft, 1977). They can be strongly altered by presence of liquid water, which may be allowed in occasional environments at the lower boundaries of permafrost layers and local spots. Clifford (1993) estimated that the groundwater may exist in relatively shallow depth, a few km below the surface. It appears difficult to probe even if it exists at the depth, but fortunately direct measurement is possible on the exposed deep layers at the bottoms of craters and canyons where tentatively could exist the water running over from aquifers.

A recent report of discovery of evidence of past life in meteorite, ALH84001, appeals the importance of exploring lives and places they existed or still exists (McKay et al., 1996). The sites where liquid water may exist should be most suitable for in situ experiments and sample-returns for exobiology. HF radio sounding would play an important role for this purpose. 
Table 1. Electrical properties of likely Martian surface materials.

\begin{tabular}{lccc}
\hline Materials & $\begin{array}{c}\text { Dielectric } \\
\text { permittivity }\end{array}$ & $\begin{array}{c}\text { DC electrical } \\
\text { conductivity [S/m] }\end{array}$ & Loss tangent \\
\hline Regolith, basaltic & $1.5-3.5$ & $10(-12) \sim 10(-15)$ & $0.008-0.1$ \\
Regolith, andesitic & $1.5-3.5$ & $10(-12) \sim 10(-15)$ & $0.001-0.01$ \\
Ice-saturated Regolith & $3-5$ & $10(-4) \sim 10(-6)$ & $0.01-0.05$ \\
Breccia, basaltic & $4-7$ & $10(-5) \sim 10(-10)$ & $0.01-0.05$ \\
Rocks, basaltic & $8-12$ & $10(-6) \sim 10(-10)$ & $0.01-0.1$ \\
Rocks, andesitic & $6-9$ & $10(-6) \sim 10(-12)$ & $0.003-0.01$ \\
Partial-melting/Moist Clay & $5-100$ & $10(0) \sim 10(-2)$ & $0.1-1.0$ \\
\hline
\end{tabular}

The characteristics of the echo intensities and temporal profiles of the Planet-B/PWS altimeter to discuss these problems is examined in the following sections.

\section{Overviews of PWS/ALT System}

As the idea and instrumental techniques of the altimeter have been proposed and introduced by Oya and Ono (1998), the PWS system is composed of 4 wire-antennas together with preamplifiers, a transmission and receiving system, and data handling system. In ALT mode of the PWS system, the center frequency is planned to be selected at $9 \mathrm{MHz}(33 \mathrm{~m}$ in wavelength), several times higher than the predicted plasma frequency of the Martian ionosphere. The transmitter peak power is up to $600 \mathrm{~W}$ and the duration of the pulsewidth is $300 \mu \mathrm{s}$ (Ono et al., 1998). The pulse repetition rate is $8 \mathrm{~Hz}$, then the altimeter experiments will be carried out every $250 \mathrm{~ms}$ to measure the topography and reflectance distribution of the Martian surfaces, corresponding to about $1 \mathrm{~km}$ along-the-track at the surface nadir points. By use of traveltime and AM phase detection method, it is expected to improve the topographic resolution up to $20 \mathrm{~m}$ (Oya and Ono, 1998).

\section{Electrical Properties of Martian Surface Layers}

The radar echo intensities provide information on the surface physical properties such as bulk density, amount of waters and ferromagnetic minerals, and maybe the subsurface structures. The electrical properties of the uppermost layers of Martian surface obtained so far from the earth-based radar observations with higher frequencies appear to be more variable than for the Moon, with dielectric constants ranging from 1.5 to 5 (Pettengill et al., 1973). This reveals the regional variation of the surface physical properties. Olhoeft and Strangway (1974) discussed the electrical properties for the regolith layers in terms of water under the Martian thermal conditions. Their results indicate that the mean surface temperature of $213 \mathrm{~K}$ at even equatorial region is low enough to disactivate any amount of water so far as electrical properties are concerned. However, surface pressures and temperatures observed by Mariner 9 occasionally became high enough to allow liquid water, in which case the electrical properties of the Martian surface could be drastically altered, even though this would be a highly local phenomenon.

The electromagnetic properties of materials used in this study are tabulated in Table 1, by analogy with those of terrestrial materials (e.g., Olhoeft and Strangway, 1974; Telford et al., 1990). The Martian surfaces are covered with desiccated soils, breccias, and boulders, typically basaltic composition at equatorial region, and with mixtures by icy components at higher latitude. However, discovery of andesitic rocks by the APXS in Mars pathfinder suggests the presence of more silicic regions and moist materials are also taken into account. The difference in these properties detected as the radar reflectivity would indicate some geological information such as basaltic to andesitic, soils to exposed rocks or ice-saturates, desiccated to moist.

\section{Radar Echo Intensities from Surface and Sub- surface}

The PWS/ALT will perform the altimeter experiments with a much longer wavelength than that of the earth-based radar observations. Echoes from Martian surface provide information on the average electrical properties to a few $10 \mathrm{~m}$ depth, without disturbance by any surface scatters like rocks and boulders. Another importance is the high transparency for the highly desiccated or inactive surface materials, since $\mathrm{HF}$ radio pulses are estimated to penetrate into several $100 \mathrm{~m}$ depth or more (e.g., Campbell and Ulrichs, 1969; Strangway and Olhoeft, 1977; Phillips et al., 1973).

The radar echo intensities from two-layered surface which is a typical case such as regolith and basement rock were calculated in this article. Although the dielectric constant varies with depth in the regolith layer, in perticular several centimeters variation at most, it is valid to be assumed constant in the layer for a long wavelength radar. The effective radar reflectivity $\rho$ is approximately introduced as a plain wave propagation into two-layered medium. The very thin and thick surface layers relative to the wavelength are considered in order to discuss briefly.

Thin surface layers are suitable to consider the influence of surface regolith and maybe a permafrost layer beneath the moist clay. For a thin surface layer as $X \leq 10 \mathrm{~m}$, the 


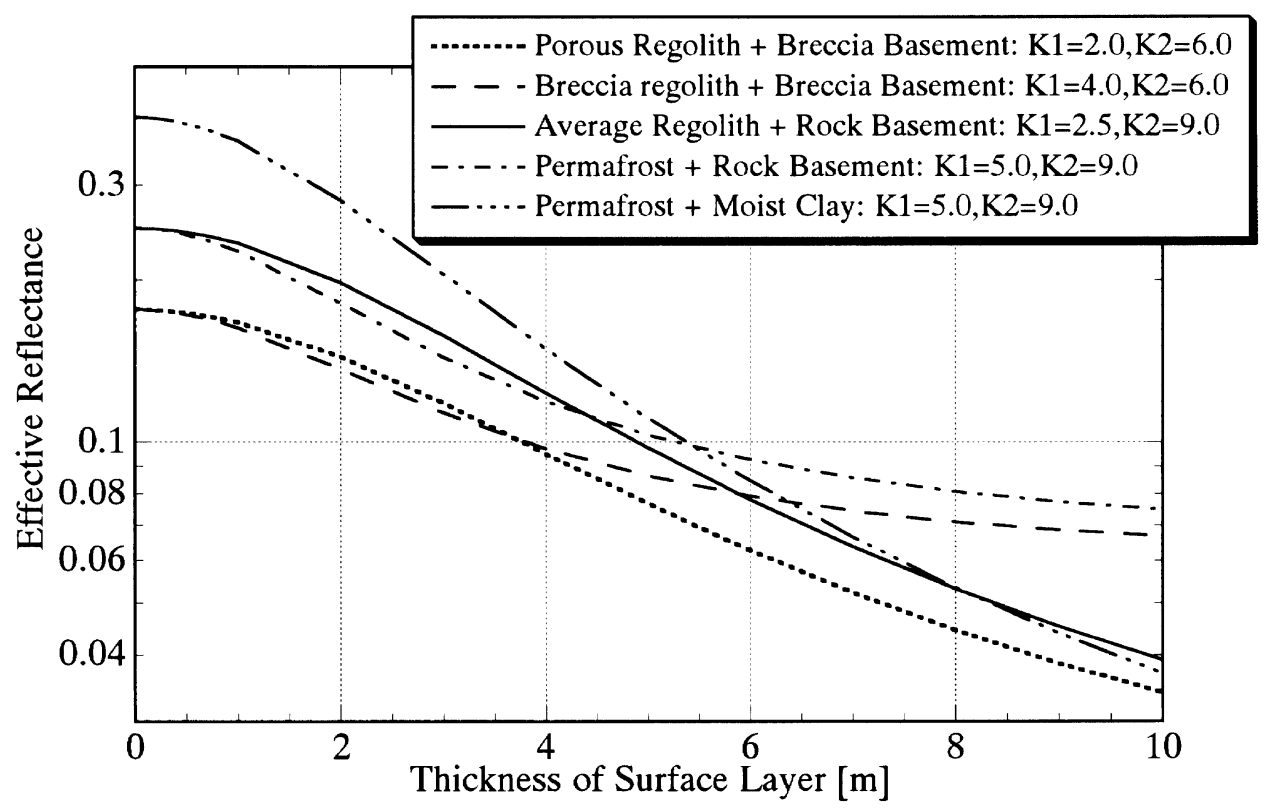

Fig. 1. Calculations of effective reflectance of two-layered surfaces with thin surface condition for various physical properties, as the function of layer depth. Surface materials and electromagnetic properties are assumed as shown in the frame. $K_{1}$ and $K_{2}$ are dielectric constants for surface and subsurface layer, respectively.

Table 2. Surface structures and electromagnetic properties.

\begin{tabular}{lccc}
\hline \multicolumn{1}{c}{ Materials and structures } & $K_{1}$ & $K_{2}$ & $D$ \\
\hline Basaltic,regolith + basement(1) & 3.0 & 10.0 & 0.1 \\
Basaltic,regolith + basement(2) & 3.0 & 10.0 & 0.03 \\
Andesitic,regolith + basement(1) & 3.0 & 7.0 & 0.01 \\
Andesitic,regolith + basement(2) & 3.0 & 7.0 & 0.003 \\
Permafrost + moist clay(1) & 5.0 & 20.0 & 0.05 \\
Permafrost + moist clay(2) & 5.0 & 20.0 & 0.01 \\
\hline
\end{tabular}

reflectivity can be expressed in an approximated form as,

$$
\begin{aligned}
& \rho \approx \\
& \left|\frac{\left(1-\sqrt{K_{2}}\right)-i \cdot \frac{2 \pi X}{\lambda}\left(1+\frac{i}{2}\left(\frac{\sigma}{K_{1} \omega}+D\right)\right) \cdot\left(\sqrt{K_{2}}-K_{1}\right)}{\left(1+\sqrt{K_{2}}\right)-i \cdot \frac{2 \pi X}{\lambda}\left(1+\frac{i}{2}\left(\frac{\sigma}{K_{1} \omega}+D\right)\right) \cdot\left(\sqrt{K_{2}}+K_{1}\right)}\right|^{2},
\end{aligned}
$$

where $K_{1}$ and $K_{2}$ are relative dielectric permittivity averaged in the surface and subsurface layers, $\sigma$ and $D$ are $D C$ electrical conductivity and loss tangent of the surface layer, $\lambda$ is the radar wavelength, $X$ is the thickness of the surface layer. So far as a desiccated surface is considered negligibly low in electrical conductivity and loss tangent, it becomes equal to the desiccated surface given in the equation introduced by Giraud (1965). It seems that the surface with much thinner or much thicker layer than wavelength reflects rader echo as if a single-layered surface with dielectric constant of $K_{2}$ or $K_{2} / K_{1}^{2}$.

Five kinds of surface models are assumed: (i) very porous regolith beneath breccia basement with $K_{1}=2.0$ and $K_{2}=$ 6.0 , (ii) similar to (i) but breccia-based or compacted regolith,
4.0 and 6.0 , respectively, (iii) regolith moderately mixed with breccia beneath rock basement, 2.5 and 9.0 respectively, (iv) similar to (iii) but saturated with ice in the upper layer, 5.0 and 9.0, respectively, and (v) moist clay below ice-saturated permafrost, 5.0 and 20.0, respectively.

Figure 1 shows the effective reflectance of radar pulses of $9 \mathrm{MHz}$ as a function of surface layer thickness for the five surface models. The surface layer of only a few meter thickness affects the reflectance and the influence is more remarkable for larger $K_{2} / K_{1}$, the reason why the surface reflection wave is more interfered with the subsurface reflection wave. The reflectance also depends on the surface material and condition. It is difficult to determine both the composition and the structure of surface at the same time. However, it becomes possible to constrain them with some assumption of materials and conditions. This suggests that the altimeter experiments can constrain the packing factor or bulk density of the regolith and its thickness which is important to solve the origin of rampart craters and to examine the stealth regions.

On the other hand, considering the much thicker surface 


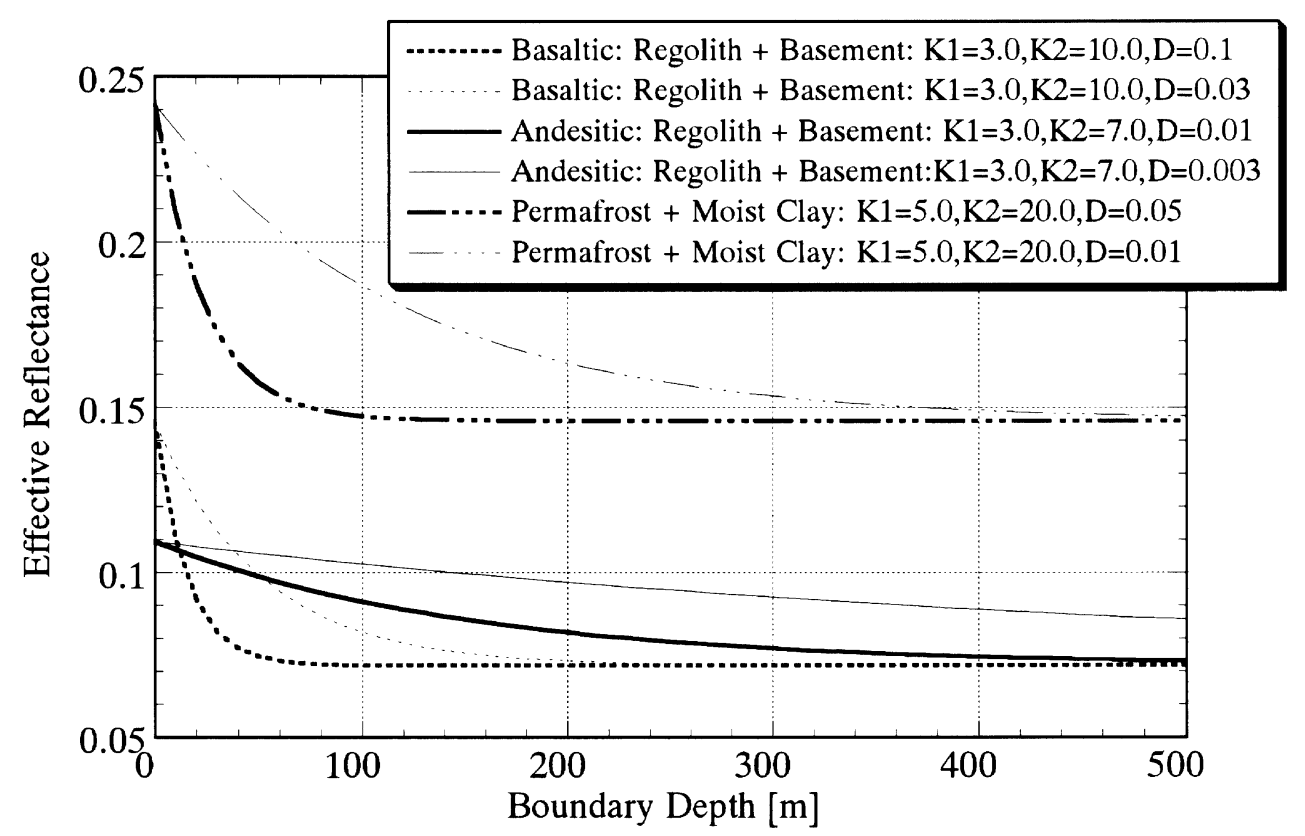

Fig. 2. Similar to Fig. 1 but for thick surface layer cases. The layer materials and structures are also shown in the frame. $K_{1}$ and $K_{2}$ are the same as Fig. 1 and $D$ means loss tangent of the surface layer.

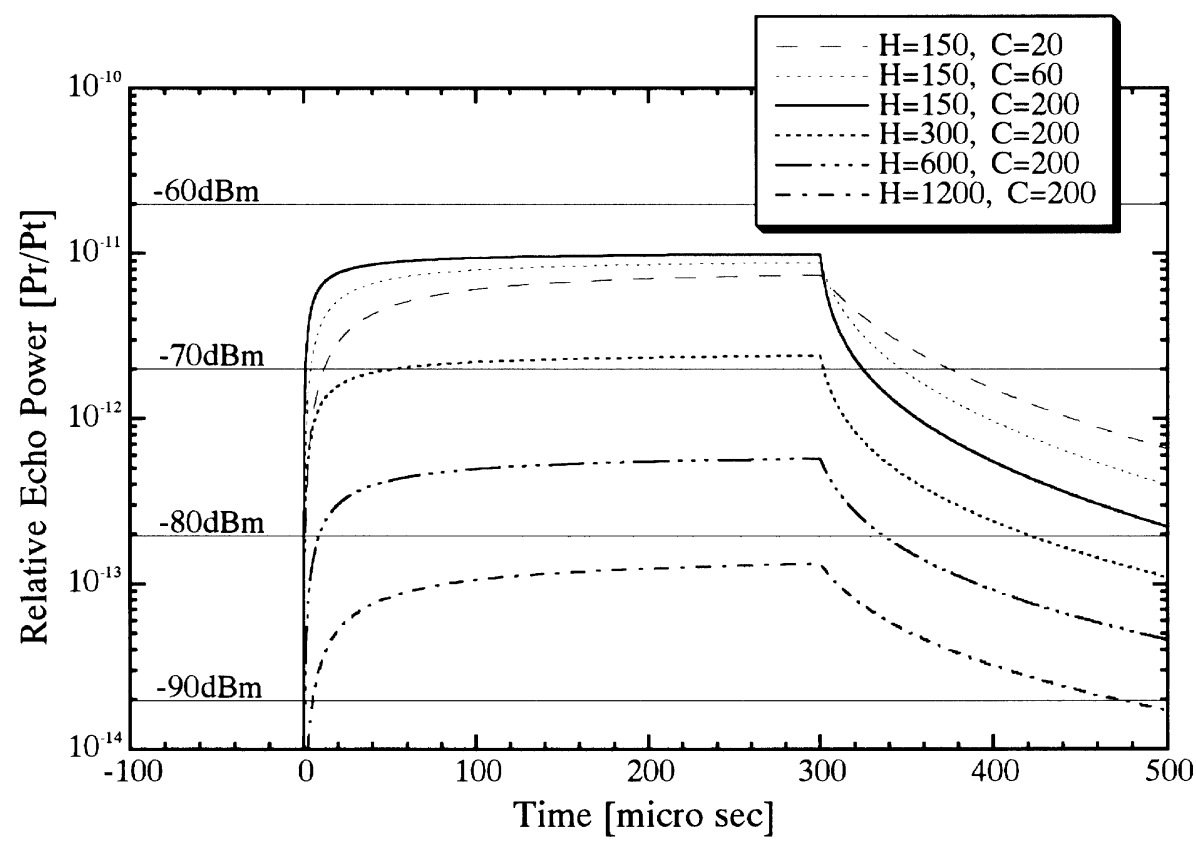

Fig. 3. Calculations of the echo profiles with parameters written in the frame. $C$ is the indicator of surface rms roughness and the rougher surface has the smaller value, i.e. $C=20$ is typical for the lunar highland and $C=200$ is typical for the lunar maria. $H$ means the orbit height in km.

layers than the radar wavelength, the reflectivity can be expressed:

$$
\rho \approx R_{1}+R_{12} \cdot T_{1}^{2} \cdot \exp \left(-\frac{2 \sigma}{\sqrt{K_{1}}} X-\frac{4 \pi D \sqrt{K_{1}}}{\lambda} X\right)
$$

where $R_{1}$ and $R_{12}$ are reflectivity at the surface and the subsurface boundary, and $T_{1}=1-R_{1}$. The reflection takes place as if a single-layered medium for increasing $X$ to in- finity. Then we assume models of regolith together with basement structures with basaltic and andesitic composition and structure of moist layers below the icy permafrost with proper electromagnetic properties as shown in Table 2.

Figure 2 shows the effective reflectance versus depth of boundary layer calculated with the surface models in Table 2 . The increasing trend of reflectance for shallower boundary depth reveals the influence of the subsurface reflectance. For 

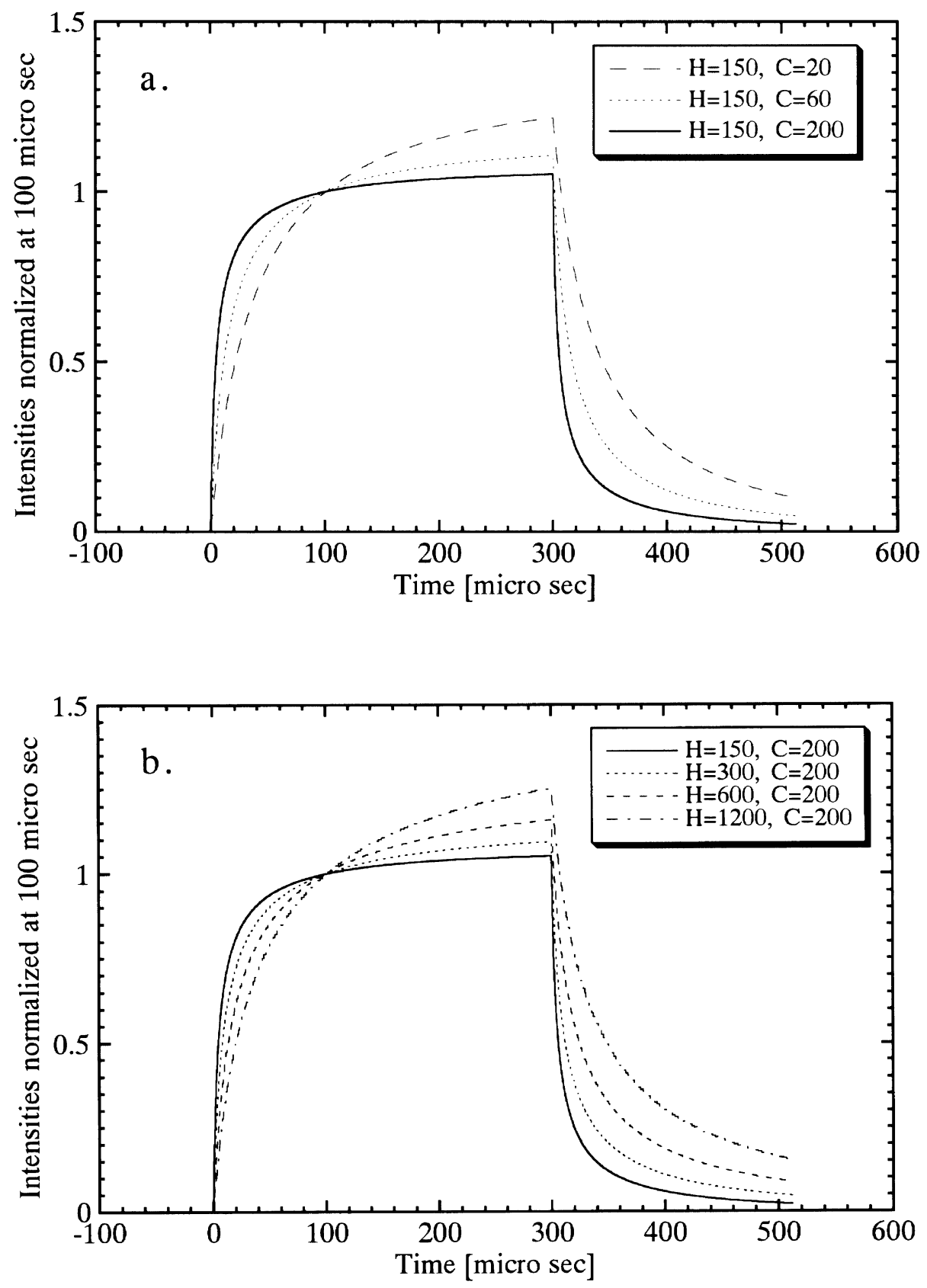

Fig. 4. The radar echo profiles are normalized to set to unity at 100 micro sec and the rise-time profiles can be compared for dependence of (a) surface roughness and (b) orbit height.

basaltic composition, that is $D \geq 0.01$, the echo from subsurface ranging tens to a hundred meter deep is detectable as the enhanced reflectance. For more silicic, andesitic, composition with $D \leq 0.01$, detection of the subsurface echo from hundreds to even a few kilometers deep is expected. Since the penetration depth of radar is mainly dependent on the loss tangent of the material in the upper layer, the probing depth of the subsurface by this method is limited up to about $100 \mathrm{~m}$ for typical, basaltic, composition, in which depth the long-term existence of liquid water is unlikely. The places where water exists would be very local sites and if exists, it would be a transient phenomenon, but the exposed subsurface layers such as bottom of craters or valleys would be the candidate places. Another candidate may happen at the lower, or warmer, end of icy saturated layer, for which the effective reflectance is also calculated as shown in Fig. 2. When the moist layer exists within hundreds of meter depth below the permafrost layer, it can be directly observed as enhanced reflectance, although there are much uncertainties of the electromagnetic properties of ice-saturated materials.

\section{Effect of Surface Roughness}

Since not a small part of Martian surfaces are disturbed by impact craterings, volcanisms and tectonics, it should be noted that the echoes of HF radio pulses are influenced by geomorphologic features even though the surface rocks and boulders are of less importance as scatters. The effect of surface roughness is discussed to obtain the real value of 
the radar normal reflectivity which offers information on the physical properties as discussed in the previous sections.

Intensities of total echo, the sum of surface and subsurface reflection are considered in this paper. Therefore, only two parameters $\rho$ and $C$, are adopted to represent the radar echo by using Hagfors' equation (Hagfors, 1970), where $\rho$ is the reflectivity of the surface materials and $C$ is a function of surface root-mean-square roughness of the target area. Radar echo intensity from a surface point with the area of $d A$ can be expressed in the differential form with an antenna-centered spherical coordinate system: $(r, \theta, \phi)$,

$$
\begin{aligned}
& d \operatorname{Pr}(\theta, t) \\
& =\frac{G^{2} \lambda^{2}}{(4 \pi)^{3} R(\theta, \phi)^{4}} \operatorname{Pt}(\theta, \phi, t-2 R(\theta, \phi) / c) \cdot \sigma_{0}(\theta) d A,
\end{aligned}
$$

where $d P r$ is the differential echo intensity, $P t$ is the transmission radar power, $G$ is the antenna gain, $\lambda$ is the wavelength of $33 \mathrm{~m}, R$ is the distance from the orbiter to a ground point, $c$ is the light velocity, $\sigma_{0}$ is the radar cross-section per unit area of Hagfors' form which is widely known to fit the observation data for various wavelength as follows,

$$
\sigma_{0}(\theta)=0.5 \rho C\left(\cos ^{4} \theta+C \sin ^{2} \theta\right)^{-3 / 2} .
$$

Using these equations, the temporal profiles of radar echo under various conditions can be obtained by integration around all the surfaces below. The compilation in terms of surface roughness and orbit altitude of these results are shown in Fig. 3, and their rise-time profiles by normalizing the original echo profiles at $100 \mu$ s are shown in Figs. $4 \mathrm{a}$ and $4 \mathrm{~b}$ for dependence of surface roughness and orbit height, respectively. We notice the peak echo power, even for $H=1200 \mathrm{~km}$, is over $-85 \mathrm{dBm}$ (the effective radiation power, $P t$, is assumed equal to $+47 \mathrm{dBm}$ for $60 \mathrm{~W})$, more than $+10 \mathrm{~dB}$ above the galactic noise level at this frequency range $(-94.6 \mathrm{dBm})$. Figure $4 \mathrm{a}$ reveals that the surface root-mean-square roughness can alter the echo profiles such that the echo from rougher surfaces show the slower rise-time. The altitudes also proves to the echo profiles as shown in Fig. $4 \mathrm{~b}$, since the echo rises up slower at higher altitudes. It is concluded from these results that the echo of the altimeter is detectable from even the altitude of $1000 \mathrm{~km}$ or higher, and the radar reflectance and surface roughness can be discriminated each other if echo intensities and temporal profiles are both obtained.

\section{Summary}

The PWS/ALT experiments can obtain the topographic profiles and the reflectance distribution of the Martian surface along-the-track with a higher accuracy and a better spatial resolution than in the previous missions. In addition, the macroscopic surface roughness and the subsurface properties will be constrained to some degree. The determination of electrical properties of Martian surfaces will play an important role of understanding the physical properties of surface layers. So that the spatial variation of non-basaltic areas and volcanism, thickness of regolith layer, and candidate moist structures might be detected. Furthermore, the origin of rampart craters and the structure of stealth region can be constrained. This method of exploration is, of course, applicable to many future planetary missions.

Acknowledgments. Authors express thanks to colleagues for many useful suggestions to complete this paper. This work was supported by JSPS Research Fellowship for Young Scientists.

\section{References}

Campbell, M. J. and J. Ulrichs, Electrical properties of rocks and their significance for lunar radar observations, J. Geophys. Res., 74, 58675881, 1969.

Clark, B. C., A. K. Baird, H. J. Rose, P. Toulmin, R. P. Christian, W. C. Kelliher, A. J. Castro, C. D. Rowe, K. Kiel, and G. Huss, The Viking X-ray fluorescence experiment: Analytical methods and early results, $J$. Geophys. Res., 82, 4577-4594, 1977.

Clifford, S. M., A model for the hydrologic and climatic behavior of water on Mars, J. Geophys. Res., 98, 10,973-11,016, 1993.

Giraud, A., A note on the radio reflectivity of the lunar surface, Radio Sci. J. Res. NBS/USNC-URSI, 5, 1677-1681, 1965.

Hagfors, T., Remote probing of the Moon by infrared and microwave emissions and by radar, Radio Sci., 5, 189-227, 1970.

McKay, D. S., E. K. Gibson, K. L. Thomas-Keprta, H. Vali, C. S. Romanek, S. J. Clemet, X. D. F. Chiller, R. Maechling, and R. N. Zare, Search for past life on Mars: Possible relic biogenic activity in Martian meteorite ALH84001, Science, 273, 924-930, 1996.

Muhleman, D. O., B. J. Buler, A. W. Grossman, and M. A. Slade, Radar images of Mars, Science, 253, 1508-1513, 1991.

Olhoeft, G. R., Electrical properties of natural clay permafrost, Can. J. Earth Sci., 14, 16-24, 1977.

Olhoeft, G. R. and D. W. Strangway, Electrical properties of the surface layers of Mars, Geophys. Res. Lett., 1, 141-143, 1974.

Ono, T., A. Morioka, A. Kumamoto, H. Oya, K. Kobayashi, T. Obara, and T. Nakagawa, Plasma waves and sounder (PWS) experiment onboard the Planet-B Mars orbiter, Earth Planets Space, 50, this issue, 213-221, 1998.

Oya, H. and T. Ono, A new altimeter for Mars land shape observations utilizing the ionospheric sounder system onboard the Planet-B spacecraft, Earth Planets Space, 50, this issue, 229-234, 1998.

Pettengill, G. H., I. I. Shapillo, and A. E. E. Rogers, Topography and radar scattering properties of Mars, Icarus, 18, 22-28, 1973.

Phillips, R. J., J. F. Adams, W. E. Brown, R. E. Eggleton, P. Jackson, R. Jordan, W. I. Linlor, W. J. Peeples, L. J. Porcello, J. Ryu, G. Schaber, W. R. Sill, T. W. Thompson, S. H. Ward, and J. S. Zelenka, Apollo lunar sounder experiment, in Apollo 17 Preliminary Science Report, Chap. 22, NASA SP-330, 1973.

Rieder, R., T. Economou, H. Waenke, A. Turkevich, J. Crisp, J. Brueckner, G. Dreibus, and H. Y. McSween, Jr., The chemical composition of Martian soil and rocks returned by the mobile alpha proton X-ray spectrometer: Preliminary results from the X-ray mode, Science, 278, 1771-1774, 1997.

Strangway, D. W. and G. R. Olhoeft, Electrical properties of planetary surfaces, Phil. Trans. R. Soc. Lond., A285, 441-450, 1977.

Telford, W. M., L. P. Geldart, and R. E. Sheriff (eds.), Electrical properties of rocks and minerals, in Applied Geophysics, 2nd edition, 770 pp., Cambridge University Press, 1990.

T. Okada (e-mail: okada@planeta.sci.isas.ac.jp) and T. Ono (e-mail: ono@stpp3.geophys.tohoku.ac.jp) 